\title{
Usability Evaluation of Software Testing Based on Analytic Hierarchy Process
}

\author{
Dandan $\mathrm{HE}^{1, \text { a }}$, Can WANG ${ }^{2}$ \\ ${ }^{1,2}$ Department of information science, Dalian Institute of Science and Technology, Dalian City \\ 116052, China \\ a dlmu_ddh@126.com
}

\section{Keywords: Software Testing, Usability Evaluation, Analytic Hierarchy Process}

\begin{abstract}
In software product development process, software usability testing is essential and must be carried out in the actual work tasks and operating environment of the user. Expand the availability of this article assessment of software testing based on analytic hierarchy process (AHP), it qualitative and quantitative indicators in a unified model, to break down complex problems into component factors, in turn govern the relationship between these factors by grouping formed hierarchical structure. Determine the relative importance of various factors in the hierarchy by pairwise comparison approach. Then determine the overall decision-makers to determine the relative importance of the decision-making program overall ranking. AHP decision-makers can make decisions directly, thus greatly improving the effectiveness of software testing to assess the availability, reliability and feasibility.
\end{abstract}

\section{Introduction}

Understanding people's software is generally up to consider from a technical, it seems the more advanced technology, the higher the level, the better the system. The so-called people's understanding, not only includes the design and management personnel, but also ordinary users. Therefore proposes the availability of software problems, not only is the designer thought of a revolution, a revolution is the ordinary understanding. In software product development process, software usability testing is an indispensable part. Availability is from a human point of view software system is easy to use, efficient and satisfactory [1]. As a special kind of IT product, its availability is particularly important. Usability testing and evaluation cannot rely on made a few questionnaires, allowing users to fill in after, through simple statistical analysis to the conclusion [2]. Usability testing must be user after the actual operation, according to the results of the fulfillment of its mandate, objective analysis and evaluation. To choose a broadly representative user. Because an important requirement for the availability of software is the system should be suitable for most people to use, and so most people are satisfied. So take the test must be representative, should be representative of the majority of users.

\section{Software Testing Availability Overview}

The concept of software testing usability mainly contains the meaning of the following three aspects. First of all, ease of use and effectiveness, that the system has improved functionality and user interface to function fully compatible with, and easy for users to learn and use. Secondly, the efficiency, the user can use the system efficiently, and in a certain time frame to complete the task properly. Finally, satisfaction, the user during use of the system's subjective feelings are satisfied. Availability proposed concept changed people's awareness and understanding of human-computer interaction, user-centered user interface design concept has been widely accepted.

Usability evaluation is the systematic collection of user interface usability data and its evaluation and correction process. One of the more common and easy method for the effective implementation of the User Test, Heuristic Evaluation and Questionnaire [3-4]. 
User Test. User testing is to test some real user through to a representative designated task, the process operation from the user to complete the task, testers use to observe, record and analyze the way of user behavior and related data are summarized, found insufficient product place, and provide a basis for optimization of the product.

Heuristic Evaluation. It is a very classic usability evaluation methods. It refers to a small number of evaluators (experts in related fields such as) to carry out a systematic review of the guidelines, according to some empirical aspects of interface design interface, so to discover the usability problems. In short, the experts use a set of usability guidelines called "inspiring principle" as a guide to assess the user interface element meets these rules.

Questionnaire. User survey is to collect people's views on a particular issue, attitudes or beliefs and other information designed for a range of issues, questions and answers related to the user record for these problems. Under normal circumstances, the question questionnaire biased in favor of closed-ended questions, users only need to give a simple answer, such as making a choice from the list, or to grade level scoring way to express the extent of the system functions like .

\section{The basic principle of the Analytic Hierarchy Process}

The Analytic Hierarchy Process (AHP) is a multi-objective decision analysis method for quantitative and qualitative [5]. Central to this approach is that policy makers will experience judgment given quantization to provide a quantitative basis for decision makers in the form of more practical goals in the complex structure and the lack of necessary data. Its basic principle is to a variety of factors related to the evaluation of alternatives to the system is divided into several levels, and in various elements of the same level on the layer elements according to the criteria, pairwise comparison judgment and calculate the weight of each element of weight, according to comprehensive weight by a maximum weight principle to determine the optimal solution.

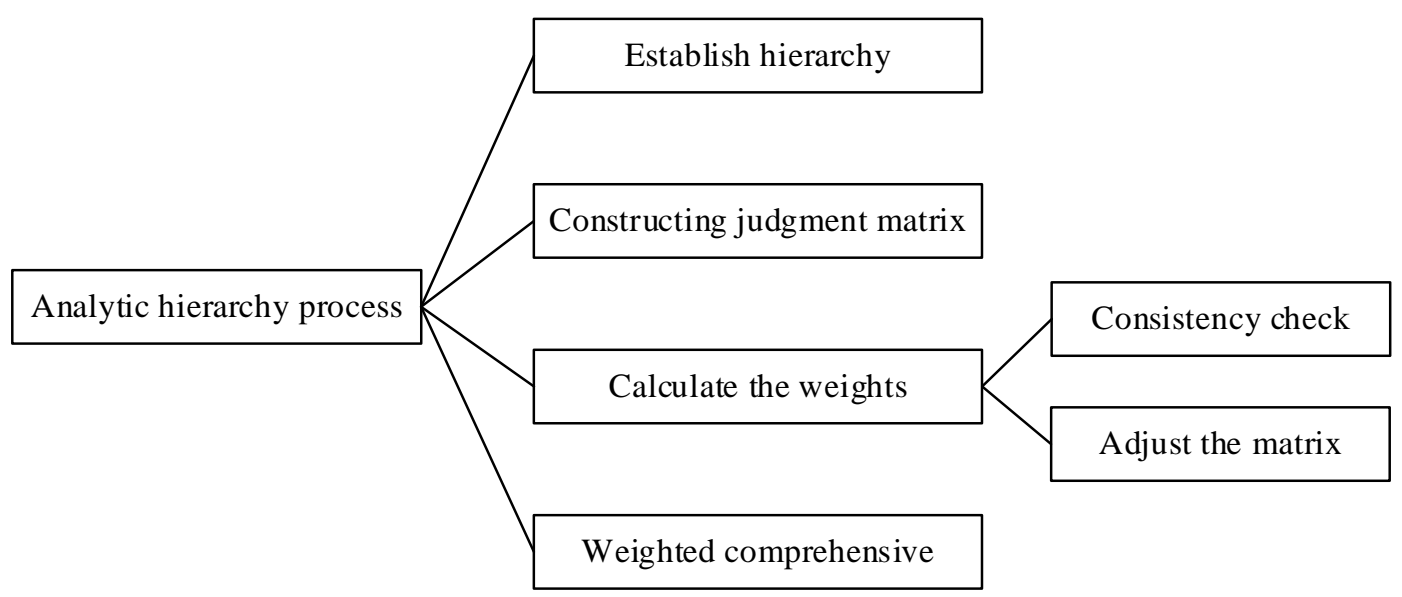

Figure 1 . The steps of analytic hierarchy process

Application analysis of decision making problems, the first to put the question a methodical, hierarchical, a hierarchy structure model is constructed. In this model, the part of the complex problem is broken into elements. These elements and forming several levels according to their attributes and relations. A hierarchy of elements as a criterion to the next level related elements reigns. The top is the target layer, it said institute to achieve goals, such as mobile Internet product availability level, or ultimately the decision to be made [6]. Summarize its basic steps roughly divided into four steps, as shown in Figure 1.

\section{Usability Evaluation of Software Testing Based on AHP}

Our proposed testability model is based on Dromey's software quality model which has been a benchmark in use for various quality features as well as many testability models so far. We have followed the steps as mentioned below to formalize the model. On the basis of our previous research work and surveys we have identified six factors to assess testability for object oriented 
software at design level. All these are internal quality characteristics - Encapsulation, Inheritance, Coupling, Cohesion, Polymorphism and Size \& Complexity. Out of six identified features four features have been proposed in MTMOOD testability model, which does not cover the polymorphism and size \& complexity feature, which have also been found as essential internal features by many researchers in testability study. These six object oriented features play a very significant role in testability improvisation directly or indirectly. The studies indicate encapsulation promotes efficiency and complexity. Inheritance has a significant influence on the efficiency, complexity, reusability and testability or maintainability [7].

But to keep study simple from AHP evaluation aspect we have chosen the few basic but popular metrics amongst testability researchers. So, the proposed testability assessment model with respect to internal design features using static metrics is as shown in Figure 2. It is based on six above mentioned object oriented features from testability perspective as pointed in Binders research too. Out of all the popular metrics suites discussed in our previous work six of these static metrics as explained below in Table2 have been identified for the evaluation of each of these feature and their effects on any object oriented software testability at design time.

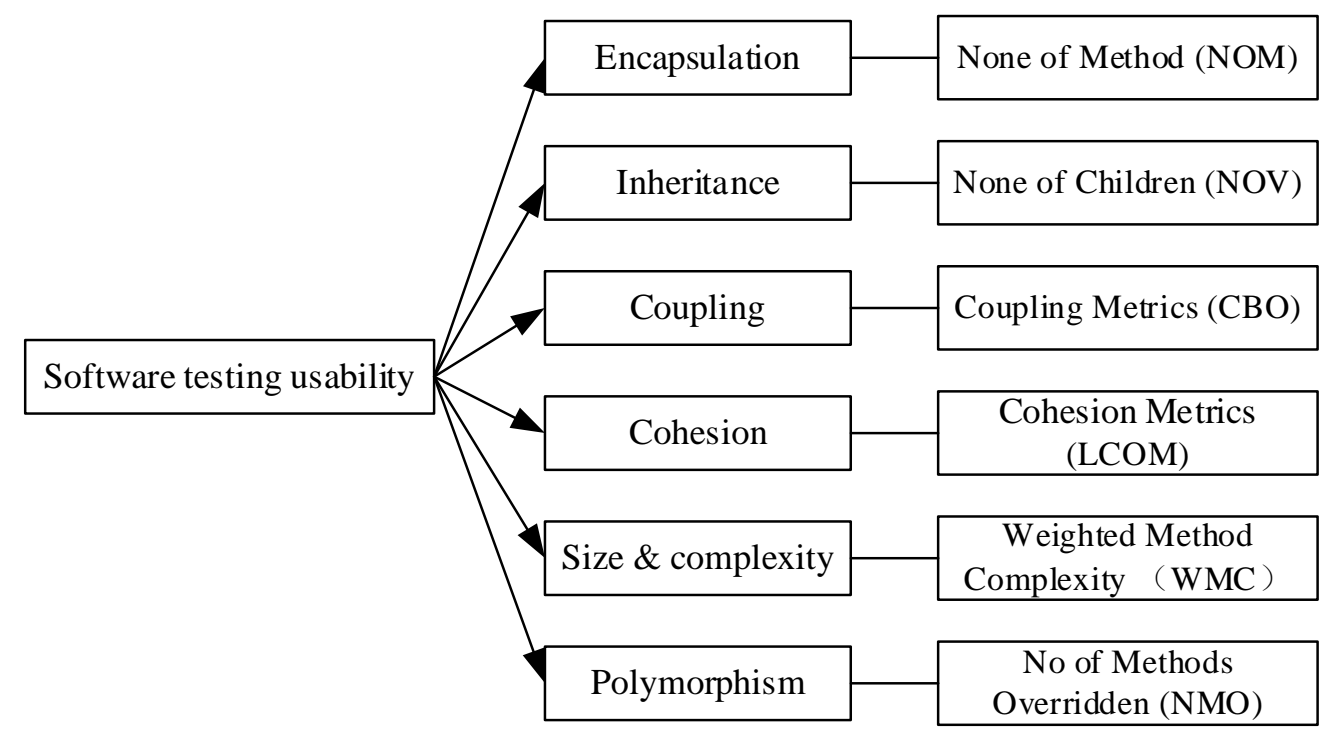

Figure 2. Proposed software testing usability assessment model with static metrics

Testability Study. In order to conduct testability study based on above model and AHP technique. The hierarchical model with factors - Encapsulation (F1), Inheritance (F2), Coupling (F3), Cohesion (F4), Size \& complexity (F5) and Polymorphism (F6). A square matrix of 6X6 is sent for pair-wise weight filling to 10 experts as discussed above. The mean matrix thus formed using these 10 samples on six testability factors is given below:

$\left[\begin{array}{ccccccc} & F 1 & F 2 & F 3 & F 4 & F 5 & F 6 \\ F 1 & 1.00 & 1.35 & 4.50 & 3.20 & 0.95 & 1.55 \\ F 2 & 1.23 & 1.00 & 4.30 & 3.30 & 1.07 & 1.40 \\ F 3 & 0.23 & 0.24 & 1.00 & 0.63 & 0.22 & 0.26 \\ F 4 & 0.32 & 0.31 & 1.95 & 1.00 & 0.27 & 0.31 \\ F 5 & 1.55 & 1.60 & 4.70 & 3.80 & 1.00 & 1.40 \\ F 6 & 0.95 & 1.10 & 3.90 & 3.30 & 1.10 & 1.00\end{array}\right]$

There are many methods for calculating the eigenvector. We have used spreadsheet based approximate calculations for local priorities of criteria. The Eigen value thus calculated are as shown in table 5 below. The eigenvector of the relative importance of F1, F2, F3, F4, F5 and F6 is $(0.22,0.22,0.04,0.06,0.25,0.20)$, which is given in Table-1. These values are weights of main factors i.e. Encapsulation (0.22), Inheritance (0.22), Coupling (0.04), Cohesion (0.06), Size \& Complexity (0.25) and Polymorphism (0.20) in testability assessment. Now the six Eigen values calculated for each of these factors is $(6.62,6.61,6.58,6.47,6.63,6.60)$ with $\lambda$ max $=6.59$ which is $>=6$ (total no of factors), which is consistent. Using this we calculate the CI and CR values as follows: 


$$
\begin{aligned}
& C I=\frac{(\lambda \max -\mathrm{n})}{n-1}=\frac{6.59-6}{6-1}=0.12 \\
& C R=C I / R I=0.12 / 1.24=0.09
\end{aligned}
$$

Table 1: Eigen vector and Eigen value for main factors

\begin{tabular}{|c|c|c|c|c|c|c|c|}
\hline & F1 & F2 & F3 & F4 & F5 & F6 & Eigen Value \\
\hline F1 & 1 & 1.35 & 4.5 & 3.2 & 0.95 & 1.55 & 0.22 \\
\hline F2 & 1.23 & 1 & 4.3 & 3.3 & 1.07 & 1.4 & 0.22 \\
\hline F3 & 0.23 & 0.24 & 1 & 0.63 & 0.22 & 0.26 & 0.04 \\
\hline F4 & 0.32 & 0.31 & 1.95 & 1 & 0.27 & 0.31 & 0.06 \\
\hline F5 & 1.55 & 1.6 & 4.7 & 3.8 & 1 & 1.4 & 0.25 \\
\hline F6 & 0.95 & 1.1 & 3.9 & 3.3 & 1.1 & 1 & 0.2 \\
\hline
\end{tabular}

We found the calculated value of $\mathrm{CR}<0.1$ in all the samples of matrices, which indicates that the estimate is consistent and acceptable. Now finally we have to construct a matrix of the eigenvectors for three selected projects P1, P2 and P3 and six testability assessment factors weights F1, F2, F3, F4, F5, and F6 as mentioned below. The overall global utility of each project is calculated using the summation of the products of the weight of OO Project with reference to each factor by the weights of corresponding factor yields the global utility of each OO Project. The best OO Project is the one which is having the highest overall testability index values. Accordingly, ranking of OO Project is done which are shown in Table 2 and P1 found to be the best choice as its testability index value is highest amongst three.

Table 2: Global overall utility and rank of all three projects testability

\begin{tabular}{|c|c|c|c|c|c|c|c|c|}
\hline & F1 & F2 & F3 & F4 & F5 & F6 & Usability & Rank \\
\hline Wj & 0.22 & 0.22 & 0.04 & 0.06 & 0.25 & 0.2 & & \\
\hline P1 & 0.62 & 0.28 & 0.69 & 0.27 & 0.62 & 0.63 & 0.52 & 1 \\
\hline P2 & 0.1 & 0.62 & 0.23 & 0.12 & 0.1 & 0.08 & 0.21 & 3 \\
\hline P3 & 0.28 & 0.1 & 0.08 & 0.61 & 0.28 & 0.29 & 0.25 & 2 \\
\hline
\end{tabular}

\section{Conclusion}

In today's constantly updated information technology, diversification of product features enhance the user during use of the complexity, usability theory this is an effective way to resolve this contradiction. In software product development process, software usability testing is essential and must be carried out in the actual work tasks and operating environment of the user. This article describes in detail how to use AHP method to software testing usability evaluation index value. The main step is to establish a hierarchical structure, construct pairwise comparison judgment matrix to calculate each element being dominated weights and consistency judgment, calculated on the weight of the overall objective of the sort, and the consistency of overall satisfaction judgment. As a practice, the paper also three software testing implementation process given the availability of evaluation data, use AHP was further elaborated, in order to achieve the purpose.

\section{References}

[1] Delice E K, Güngör Z. The usability analysis with heuristic evaluation and analytic hierarchy process[J]. International Journal of Industrial Ergonomics, 2009, 39(6): 934-939.

[2] Jadhav A S, Sonar R M. Framework for evaluation and selection of the software packages: A hybrid knowledge based system approach[J]. Journal of Systems and Software, 2011, 84(8): 1394-1407.

[3] Heo J, Ham D H, Park S, et al. A framework for evaluating the usability of mobile phones based on multi-level, hierarchical model of usability factors[J]. Interacting with Computers, 2009, 21(4): 263-275. 
[4] Jadhav A S, Sonar R M. Evaluating and selecting software packages: A review[J]. Information and software technology, 2009, 51(3): 555-563.

[5] Behkamal B, Kahani M, Akbari M K. Customizing ISO 9126 quality model for evaluation of B2B applications[J]. Information and software technology, 2009, 51(3): 599-609.

[6] Yuen K K F, Lau H C W. A fuzzy group analytical hierarchy process approach for software quality assurance management: Fuzzy logarithmic least squares method[J]. Expert Systems with Applications, 2011, 38(8): 10292-10302.

[7] Subramanian N, Ramanathan R. A review of applications of Analytic Hierarchy Process in operations management[J]. International Journal of Production Economics, 2012, 138(2): 215-241. 